\title{
Astronomia Cultural
}

Javier Mejuto ${ }^{1}$

A lo largo de la existencia del ser humano el cielo ha sido visto como referencia, como un gigantesco reloj que nos permite tener una noción temporal estable, como fuente de luz y oscuridad, como generador de vida a través del crecimiento de las plantas, por tanto, no podía más que ser un lienzo donde la humanidad ha proyectado sus deseos, sus sueños y sus mitos.

Esta relación que existe entre el ser humano y el espacio celeste dentro de su contexto cultural es el objeto de estudio de la Astronomía Cultural (Iwaniszewski 1990, 1991, 1994). Esto incluye una variedad de expresiones culturales que reflejan conocimiento astronómico: objetos, tradiciones culturales, sistemas de creencias, etcétera. A partir de estas ideas podemos inferir dos características básicas de la disciplina: es eminentemente interdisciplinar y dista del objeto de estudio de la Astronomía que -en su versión más clásica (sin incluir la Astrofísica y la Cosmología)- estudia la posición y las leyes del movimiento de los cuerpos celestes. La Astronomía Cultural estudia el registro del tiempo, los motivos textiles, la navegación, construcciones de todo tipo, motivos de arte rupestre y un largo etcétera que involucran a disciplinas como la Psicología, Arquitectura, Astronomía, Arqueología, Ciencias del patrimonio entre muchas otras.

Superada la controversia durante decenios entre los enfoques europeo y americano, en los que el primero se centraba en la metodología de medición y toma de datos y el segundo en el que se enfatizaba los aspectos culturales, se mostraba así la clásica diferenciación entre las Ciencias Naturales y las Ciencias Sociales como apartados estancos de la realidad, la disciplina entró en un enfoque mixto en el que al rigor metodológico se aúnan análisis basados en enfoques culturales. Ello proporcionó investigaciones más completas y con mayor pertinencia que despertaron el interés de los científicos sociales que, hasta ese momento, no incorporaron al cuerpo teórico de

\footnotetext{
${ }^{1}$ Profesor investigador, actualmente se desempeña como Jefe del Departamento de Arqueoastronomía y Astronomía Cultural y Coordinador del Grupo de Investigación Científica de Astronomía Cultural. Facultad de Ciencias Espaciales UNAH; javier.mejuto@unah.edu.hn
} 
sus investigaciones científicas. En la actualidad, la Astronomía Cultural es una disciplina con un fuerte crecimiento en todo el mundo, clásicamente orientada a descubrir el conocimiento astronómico que permanece registrado en los restos materiales de las culturas que habitaron las diferentes regiones culturales en el pasado. Sin embargo, en la actualidad -y cada vez con mayor fuerza- están apareciendo estudios de conocimiento astronómicos en la intersección con lo que se denominan estudios indígenas. Estos estudios poseen una mayor relación con la tradición oral, el arte, las ciencias de la religión, la literatura y la etnología ya que se ocupan de aquellos pueblos contemporáneos que poseen una cultura diferente a las predominantes occidentales. A través de estos trabajos se ha podido rescatar una gran información sobre conocimiento indígena que nos ha permitido abrir una ventana a la visión que estos pueblos tienen del mundo, es decir, de su cosmovisión. Se establece una relación biunívoca entre el espacio celeste y el espacio terrestre humano en el que se intenta representar la visión simbólica del primero en el segundo, reflejándose en todos los aspectos de la cultura. Este conocimiento se extiende en ocasiones incluso de forma diacrónica a aquellos pueblos con pasado arqueológico pero también dando visibilidad a aquellas culturas que no forman parte de un pasado común con las principales culturas de la antigüedad.

Es por lo tanto -la Astronomía Cultural- una herramienta que puede ser útil a la hora de desarrollar la identidad cultural de los pueblos originarios, otorgándoles de una visión histórica de sí mismos a través de una continuidad entre su herencia, acervo y legado cultural. Ello debe desembocar en reducir los niveles de exclusión social y en especial aquellos motivados por elementos culturales. Por otro lado -desde el punto de vista académico- la apertura de nuevas áreas del conocimiento y disciplinas permite romper el pernicioso oligopolio epistemológico que abunda en numerosos países de nuestro entorno (Derecho, Medicina e Ingeniería en el caso hondureño) estimulando a que los estudiantes y ciudadanos puedan afrontar con éxito los retos y desafíos de una sociedad tecnológica basada en el conocimiento en continuo cambio. Son estos campos interdisciplinares -como la Astronomía Cultural- los que permiten diluir los artificiales límites entre las Ciencias Sociales, Humanidades y Ciencias Naturales, integrando una visión científica integral del mundo en el que vivimos.

Como podemos ver, las implicaciones de la disciplina fuera de la academia tocan diversos ámbitos sociales; señalaré aquí solamente algunos. La enseñanza de la Astronomía Cultural en las aulas nos permite desarrollar en los alumnos (de todos los niveles) una serie de valores sociales que fomentan el desarrollo de una ciudadanía global. Además del fomento de los valores científicos típicos de la inclusión de la 
temática científica en los currículos -como son el desarrollo de un pensamiento lógico y crítico y una visión objetiva de la realidad independiente de prejuicios o gustos del observador- se incluyen valores sociales como el respeto o valoración de la diversidad, respeto por el patrimonio, importancia de la inclusión social con independencia de motivos sociales, raciales o económicos e incluso nuevos conceptos y paradigmas científicos como investigación participativa con las comunidades e investigación militante.

La relación que promueve el astrónomo cultural entre la Sociedad en general y los pueblos originarios es de gran importancia en su labor de vinculación e investigación. Se convierte en un puente entre diferentes realidades e imaginarios epistemológicos que fomenta el contacto y entendimiento entre diferentes culturas que, en numerosas ocasiones, comparten un mismo territorio o historia política. Ello puede reducir las fricciones y problemas entre comunidades que suelen producirse, en gran parte, por una falta de comprensión de la visión o intereses del otro. Por lo tanto, el astrónomo cultural no es solamente un investigador cuya labor es comprender el conocimiento astronómico que existe inextricablemente unido a las diferentes culturas sino que debe ser un nexo de unión entre culturas al comprenderlas desde un punto de vista que es compartido por todas ellas desde la antigüedad hasta nuestros días: el espacio celeste.

Mención especial merece el concepto de Patrimonio Astronómico, un concepto que han venido desarrollando durante varios años la UNESCO, ICOMOS y la Unión Astronómica Internacional (IAU) en estrecha relación (UNESCO, 2015). Este categorización patrimonial ha dado un nuevo empuje a la Astronomía Cultural permitiendo incorporarla a grupos de trabajo e investigación en ciencias del patrimonio. Esta visibilidad y enfoque también permite implementar el turismo astronómico cultural como lo realiza la propuesta alemana "Himmelswege" (Los caminos del cielo) (Sachsen-Anhalt, 2015). Sin duda este tipo de propuestas -si se realizan con el imprescindible enfoque cultural- brindan una posibilidad de empleo y desarrollo para las regiones donde el patrimonio astronómico es relevante como es el caso de Honduras.

Las salidas profesionales con las que cuentan la disciplina en la actualidad no solo incluyen a las tareas académicas si no que podemos incluir: gestor de patrimonio (astronómico), consultor arqueológico o antropológico, educador o desarrollador de currículum con pertinencia cultural, curador de museos o galerías de arte, educador astronómico con pertinencia cultural, gestor de divulgación, guía turístico, consultor turístico, comunicador o periodística científico con pertinencia cultural, desarrollador de políticas públicas con pertinencia cultural, ... 
No quisiera acabar sin comentar la labor realizada en los últimos años en Honduras y en la región en el campo de la Astronomía Cultural. En la Universidad Nacional Autónoma de Honduras a través del Departamento de Arqueoastronomía y Astronomía Cultural de la Facultad de Ciencias Espaciales se dispone del único departamento académico de la disciplina que existe en la actualidad. El departamento creado en 2009 -además de haber desarrollado un gran número de proyectos de investigación y vinculación en el campo de la Astronomía Cultural- se encuentra en proceso de aprobación de la Licenciatura en Astronomía Cultural. Una propuesta única en el mundo - y apoyada en la experiencia adquirida por más de 7 años de docencia en Astronomía Cultural con más de 800 alumnos por año académico- que permitirá formar a los futuros astrónomos culturales con el mayor rigor conceptual y metodológico dentro de un estándar común en la disciplina, un anhelo generacional en la disciplina que por fin se vuelve una realidad. De hecho, el departamento se proyectó en el futuro cercano como el mayor centro de formación en Astronomía Cultural a nivel mundial con el diseño e implementación de propuestas de posgrado especializadas en diferentes ámbitos culturales incluyendo, obviamente el centro Americano y hondureño.

Asimismo se creó en 2015 -a propuesta del Departamento de Arqueoastronomía y Astronomía Cultural y la Facultad de Ciencias Espaciales- el Instituto de Arqueoastronomía y Patrimonio Cultural y Natural (IARPACUNA), un instituto de investigación situado en Copán Ruinas y creado en colaboración con la Asociación Copán y con el apoyo de numerosas instituciones académicas y de investigación como la Universidad de Harvard y el Instituto Arqueológico Alemán (DAI).

La proyección de la disciplina en la Universidad Nacional Autónoma de Honduras a través del Departamento de Arqueoastronomía y Astronomía Cultural de la Facultad de Ciencias Espaciales ha sido destacada desde su creación. El trabajo realizado durante estos años ha permitido la internacionalización del departamento y de su labor, liderando procesos formativos que son únicos a nivel global. Creo que al ser un campo tan disciplinar, solo resta conminar al lector a introducirse en este maravilloso campo y descubrir un conocimiento común a todos los hondureños del pasado y del presente así como a los centroamericanos y humanos de todo el mundo tanto del pasado como del presente. Juntos, así podremos colaborar en crear un futuro de inclusión social basado en una educación con pertinencia cultural. 
Figura 1 y 2. Actividades de conocimiento astronómico en culturas originarias hondureñas con jóvenes Lencas y Garífunas

Figura 1

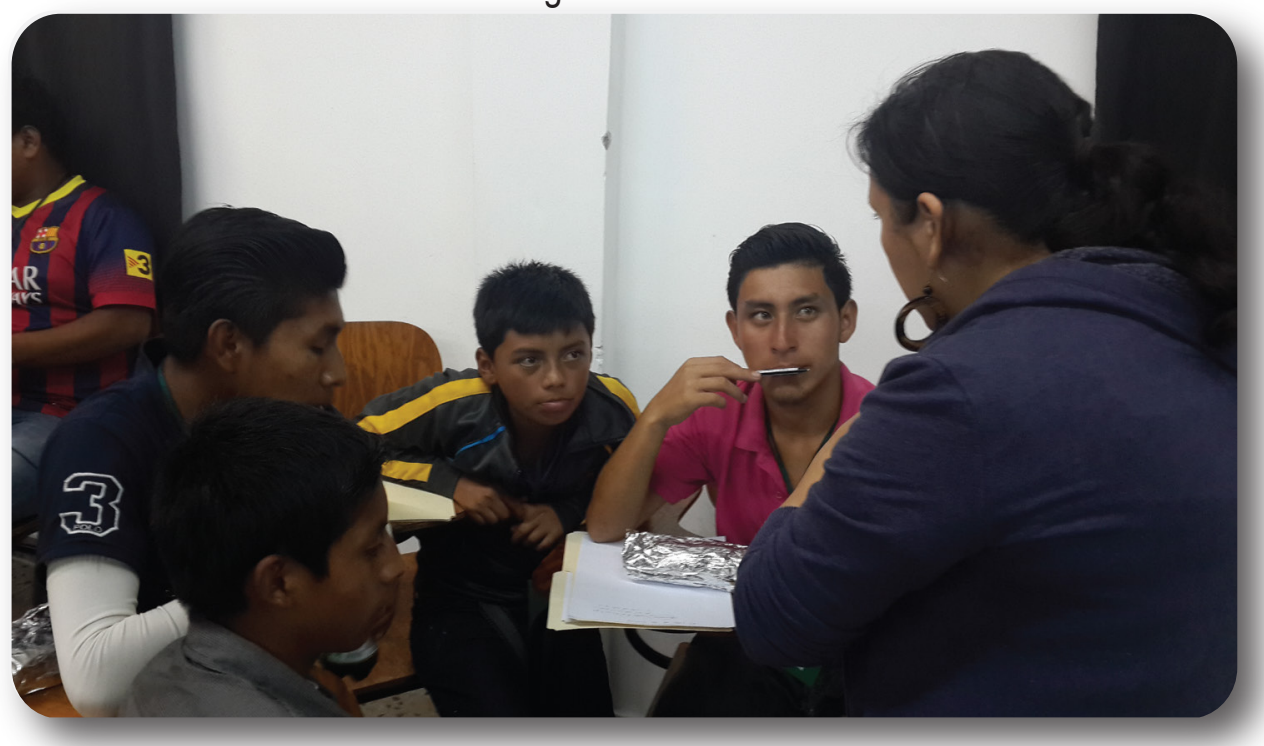

Figura 2

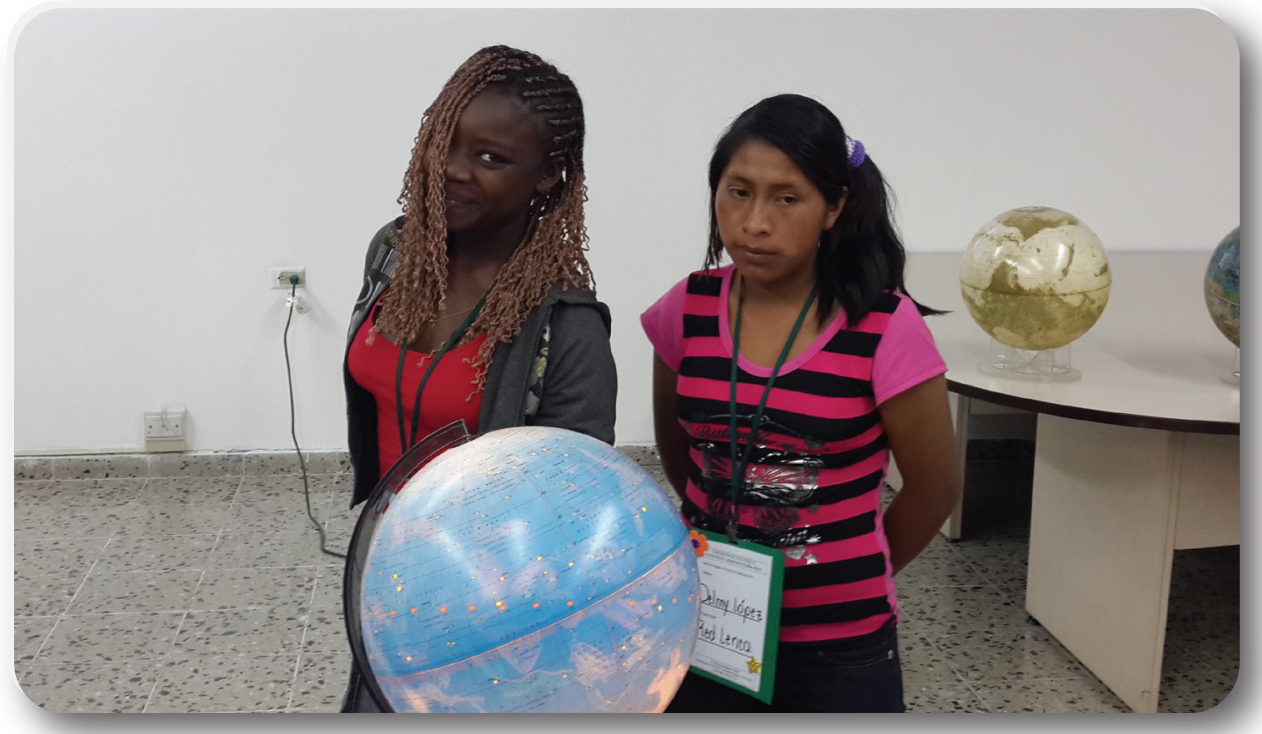

Fuente: Departamento de Arqueoastronomía y Astronomía Cultural 


\section{Bibliografía}

Iwaniszewski, S. (1990), Astronomiia kak kul'turnaia sistema, Na rubezhakh poznaniia vselennoy, A. A. Gurshtein, Moskva, Nauka: 67-73.

Iwaniszewski, S. (1991), Astronomy as a Cultural System, Interdisciplinarni izsledvaniya, 18: 282-288.

Iwaniszewski, S. (1994), De la Astroarqueología a la Astronomía Cultural, Trabajos de Prehistoria, 51(2): 5-20

Sachsen-Anhalt (2015) Himmelswege: Die archäologische Tourismusroute in Sachsen-Anhalt recuperado de http://www.himmelswege.de/en/tourist-route/nebra-ark-visitor-centre.html

UNESCO (2015) Portal to the Heritage of Astronomy recuperado de http://www2.astronomicalheritage.net/ 